\title{
PROOF OF A CONJECTURE OF P. ERDÖS ON THE DERIVATIVE OF A POLYNOMIAL
}

\author{
PETER D. LAX
}

Introduction. We start out from the following consequence of S. Bernstein's well known theorem on trigonometric polynomials. Let $p_{n}(z)$ be a polynomial of degree $n$ for which $\left|p_{n}(z)\right| \leqq 1$ holds as $|z| \leqq 1$; then $\left|p_{n}^{\prime}(z)\right| \leqq n$ as $|z| \leqq 1$ with $\left|p_{n}^{\prime}(z)\right|=n$ if and only if $p_{n}(z)=a z^{n},|a|=1$.

Some time ago P. Erdös conjectured that if $\left|p_{n}(z)\right| \leqq 1$ holds as $|z| \leqq 1$ and $p_{n}(z)$ has no roots inside the unit circle, then $\left|p_{n}^{\prime}(z)\right| \leqq n / 2$ as $|z| \leqq 1$. In the present note we give a proof of this conjecture.

Preliminaries. Let us introduce the following notation which shall be used throughout this paper:

$$
\begin{array}{ll}
p_{n}(z)=c \prod_{\nu=1}^{n}\left(z-z_{\nu}\right), & c \neq 0 ; \\
q_{n}(z)=c \prod_{\nu=1}^{n}\left(1-z \bar{z}_{\nu}\right)=z^{n} \bar{p}_{n}\left(z^{-1}\right) . &
\end{array}
$$

Then for $|z|=1$ we have $\left|p_{n}(z)\right|=\left|q_{n}(z)\right|$.

LEMMA I. If $p_{n}(z)$ has no roots inside the unit circle, that is $\left|z_{\nu}\right| \geqq 1$, the polynomial $p_{n}(z)+\epsilon q_{n}(z),|\epsilon|=1$, will have all its roots on the unit circle. ${ }^{1}$

LEMma II. If $p_{n}(z)$ has no roots inside the unit circle, $\left|z_{\nu}\right| \geqq 1$, we have $\left|p_{n}^{\prime}(z)\right| \leqq\left|q_{n}^{\prime}(z)\right|$ as $|z|=1$.

Let $z \neq z_{\nu}$; using the abbreviation $z^{-1} z_{\nu}=A_{\nu}$ we find

$$
\begin{aligned}
& \left|p_{n}^{\prime}(z) / p_{n}(z)\right|=\left|\sum_{\nu=1}^{n}\left(z-z_{\nu}\right)^{-1}\right|=\left|\sum_{\nu=1}^{n}\left(1-A_{\nu}\right)^{-1}\right|, \\
& \left|q_{n}^{\prime}(z) / q_{n}(z)\right|=\left|\sum_{\nu=1}^{n}\left(z-\bar{z}_{\nu}^{-1}\right)^{-1}\right|=\left|\sum_{\nu=1}^{n} A_{\nu}\left(1-A_{\nu}\right)^{-1}\right| .
\end{aligned}
$$

Since $\left|A_{\nu}\right| \geqq 1, A_{\nu} \neq 1$, we obtain

Received by the editors March 8, 1944.

1 G. P6lya and G. Szegö, Aufgaben und Lehrsätze aus der Analysis, Berlin, 1925, vol. 1, p. 88, Problem 26. 
(1)

$$
\begin{gathered}
\Re\left(1-A_{\nu}\right)^{-1} \leqq 1 / 2, \quad \Re \sum\left(1-A_{\nu}\right)^{-1} \leqq n / 2, \\
\left|\Re \sum\left(1-A_{\nu}\right)^{-1}\right| \leqq\left|\Re \sum\left(1-A_{\nu}\right)^{-1}-n\right|=\left|\Re \sum A_{\nu}\left(1-A_{\nu}\right)^{-1}\right| .
\end{gathered}
$$

On the other hand

$$
\left|\Im \sum\left(1-A_{\nu}\right)^{-1}\right|=\left|\Im \sum\left(1-A_{\nu}\right)^{-1} A_{\nu}\right| \text {. }
$$

(1) and (2) prove Lemma II.

Proof of the special case, when all the roots are on the unit circle. The first proof for this special case was supplied by Professor Szegö. His argument is as follows.

Let $\left|z_{\nu}\right|=1$ and $\max \left|p_{n}^{\prime}(z)\right|=\left|p_{n}^{\prime}\left(z_{0}\right)\right|,\left|z_{0}\right|=1$.

Case I. $p_{n}\left(z_{0}\right) \neq 0$. Since $p_{n}^{\prime}\left(z_{0} e^{i \theta}\right) p_{n}^{\prime}\left(\bar{z}_{0} e^{-i \theta}\right)$ is maximum for $\theta=0$, we find that $z_{0} p_{n}^{\prime \prime}\left(z_{0}\right) / p_{n}^{\prime}\left(z_{0}\right)$ is real. Now

$$
\begin{aligned}
z p_{n}^{\prime}(z) / p_{n}(z) & =\sum_{v=1}^{n} z /\left(z-z_{v}\right), \\
z^{2} p_{n}^{\prime \prime}(z) / p_{n}(z) & =\left(\sum_{v=1}^{n} z /\left(z-z_{v}\right)\right)^{2}-\sum_{\nu=1}^{n}\left(z /\left(z-z_{\nu}\right)\right)^{2}, \\
z p_{n}^{\prime \prime} / p_{n}^{\prime}(z) & =\sum_{v=1}^{n} z /\left(z-z_{v}\right)-\sum_{\nu=1}^{n}\left(z /\left(z-z_{v}\right)\right)^{2} / \sum_{\nu=1}^{n} z /\left(z-z_{\nu}\right) .
\end{aligned}
$$

Let $z_{0} /\left(z_{0}-z_{v}\right)=1 / 2+i t_{\nu} ;$ then

$$
z_{0} \frac{p_{n}^{\prime \prime}\left(z_{0}\right)}{p_{n}^{\prime}\left(z_{0}\right)}=n / 2+i \sum t_{\nu}-\frac{\sum\left(1 / 4-t_{\nu}^{2}\right)+i \sum t_{\nu}}{n / 2+i \sum t_{\nu}}
$$

so that

$$
\sum t_{\nu}=\frac{(n / 2) \sum t_{\nu}-\sum t_{\nu} \sum\left(1 / 4-t_{\nu}^{2}\right)}{(n / 2)^{2}+\left(\sum t_{v}\right)^{2}}
$$

and

$$
z_{0} \frac{p_{n}^{\prime \prime}\left(z_{0}\right)}{p_{n}^{\prime}\left(z_{0}\right)}=n / 2-\frac{(n / 2) \sum\left(1 / 4-t_{v}^{2}\right)+\left(\sum t_{v}\right)^{2}}{(n / 2)^{2}+\left(\sum t_{v}\right)^{2}} .
$$

Case Ia. $\sum t_{p}=0$,

$$
z_{0} p_{n}^{\prime}\left(z_{0}\right) / p_{n}\left(z_{0}\right)=n / 2+i \sum t_{\nu}=n / 2,\left|p_{n}^{\prime}\left(z_{0}\right)\right|=(n / 2)\left|p_{n}\left(z_{0}\right)\right| \leqq n / 2 \text {. }
$$

Case Ib. $\sum t_{\nu} \neq 0$ so that from (3),

$$
(n / 2)^{2}+\left(\sum t_{\nu}\right)^{2}=n / 2-\sum\left(1 / 4-t_{v}^{2}\right) .
$$


Hence from (4),

$$
\begin{aligned}
z_{0} \frac{p_{n}^{\prime \prime}\left(z_{0}\right)}{p_{n}^{\prime}\left(z_{0}\right)} & =n / 2-\frac{(n / 2)^{2}-(n / 2)^{3}-(n / 2)\left(\sum t_{v}\right)^{2}+\left(\sum t_{v}\right)^{2}}{(n / 2)^{2}+\left(\sum t_{v}\right)^{2}} \\
& =n / 2+n / 2-1=n-1 ; \\
\left|p_{n}^{\prime}\left(z_{0}\right)\right| & =(n-1) \max \left|p_{n}^{\prime}(z)\right| .
\end{aligned}
$$

According to S. Bernstein's theorem this is possible only when $\left|p_{n}^{\prime}(z)\right|=$ const $z^{n-1}$ or $p_{n}(z)=$ const $\left(z^{n}+c\right),|c|=1$.

Case II. $p_{n}\left(z_{0}\right)=0$. Since $p_{n}^{\prime}\left(z_{0}\right) \neq 0, z_{0}$ is a simple root of $p_{n}(z)$, say $z_{0}=z_{\mu}$. Now

$$
\begin{aligned}
z_{0} \frac{p_{n}^{\prime}\left(z_{0}\right)}{p_{n}^{\prime}\left(z_{0}\right)} & =\lim _{z \rightarrow z_{\mu}} z \frac{p_{n}^{\prime \prime}(z)}{p_{n}^{\prime}(z)}=\lim _{z \rightarrow z_{\mu}} \frac{\left(\sum z /\left(z-z_{\nu}\right)\right)^{2}-\sum\left(z /\left(z-z_{\nu}\right)\right)^{2}}{\sum z /\left(z-z_{\nu}\right)} \\
& =2 \sum_{\nu \neq \mu} z_{\mu} /\left(z_{\mu}-z_{\nu}\right)=n-1+2 i \sum_{\nu \neq \mu} t_{\nu}
\end{aligned}
$$

which is real, consequently equal to $n-1$; see case Ib.

Second proof of the previous special case. A much simpler proof is due to Professor G. Pólya. Let $c=|c| e^{i \gamma}, z_{\nu}=e^{i \theta_{\nu}}$; then

$$
\begin{aligned}
h(\theta) & =i^{n} e^{-i \gamma} \prod_{\nu=1}^{n}\left(e^{-i \theta_{\nu} / 2}\right) \cdot e^{-i n \theta} p_{n}\left(e^{2 i \theta}\right) \\
& =|c| \prod_{\nu=1}^{n}\left[i e^{i\left(\theta-\theta_{\nu} / 2\right)}-i e^{-i\left(\theta-\theta_{\nu} / 2\right)}\right]
\end{aligned}
$$

is a trigonometric polynomial of degree $n$ with real coefficients. Hence according to a sharper form of S. Bernstein's theorem ${ }^{2}$

$$
\begin{aligned}
& \left(h^{\prime}(\theta)\right)^{2}+n^{2}(h(\theta))^{2} \leqq n^{2}, \quad\left|h^{\prime}(\theta)+i n h(\theta)\right| \leqq n, \\
& \left|d p_{n}\left(e^{2 i \theta}\right) / d \theta\right| \leqq n, \quad 2\left|p_{n}^{\prime}(z)\right| \leqq n, \quad|z|=1 .
\end{aligned}
$$

Proof in the general case. Let $p_{n}(z)$ be a polynomial of degree $n$, $\left|p_{n}(z)\right| \leqq 1$ for $|z| \leqq 1, p_{n}(z) \neq 0$ for $|z|<1$. We define $Q_{n}(z)=\left(p_{n}(z)\right.$ $\left.+\epsilon q_{n}(z)\right) / 2$, and determine $\epsilon$ so that $|\epsilon|=1, \epsilon q_{n}^{\prime}(z) / p_{n}^{\prime}(z)$ should be real and positive at $z=z_{0},\left|z_{0}\right|=1$, where $\left|p_{n}^{\prime}(z)\right|$ reaches its maximum.

Then $\left|Q_{n}(z)\right| \leqq 1,|z| \leqq 1$.

Also, by Lemma I, $Q_{n}(z)$ has all its roots on the unit circle.

2 Inequality (5) can be found in J. G. van der Corput and G. Schaake, Ungleichungen fiur Polynome und trigonometrische Polynome, Compositio Math. vol. 2 (1935) p. 337; it follows from an older and sharper theorem of G. Szegö, Über einen Satz des Herrn Serge Bernstein, Schriften der Königsberger Gelehrten Gesellschaft vol. 22 (1928) p. 69. 
We have, by Lemma II,

$$
\begin{aligned}
\max _{|z|=1}\left|Q_{n}^{\prime}(z)\right| & \geqq\left|Q_{n}^{\prime}\left(z_{0}\right)\right|=\left|p_{n}^{\prime}\left(z_{0}\right)+\epsilon q_{n}^{\prime}\left(z_{0}\right)\right| / 2 \\
& =\left|q_{n}^{\prime}\left(z_{0}\right)\right| / 2+\left|p_{n}^{\prime}\left(z_{0}\right)\right| / 2 \\
& \geqq\left|p_{n}^{\prime}\left(z_{0}\right)\right|=\max _{|z|=1}\left|p_{n}^{\prime}(z)\right| .
\end{aligned}
$$

But max $\left|Q_{n}^{\prime}(z)\right| \leqq n / 2$, therefore the same follows for $p_{n}(z)$. This establishes the conjecture of P. Erdös. The bound is obviously the best possible, as the example $\left(1+z^{n}\right) / 2$ shows.

REMARK. Returning to the case when all the roots $z_{\nu}$ of $p_{n}(z)$ are on the unit circle, it is interesting to observe that in this particular case $\max \left|p_{n}^{\prime}(z)\right|=(n / 2) \max \left|p_{n}(z)\right|=n / 2,|z| \leqq 1$. Indeed, let us write $\left|p_{n}\left(z^{\prime}\right)\right|=\max \left|p_{n}(z)\right|,|z| \leqq 1$; then $\max \left|p_{n}^{\prime}(z)\right| \geqq\left|p_{n}^{\prime}\left(z^{\prime}\right)\right|$ $=\left|p_{n}\left(z^{\prime}\right)\right|\left|\sum_{v=1}^{n}\left(z-z_{v}\right)^{-1}\right| \geqq\left|p_{n}\left(z^{\prime}\right)\right|\left|\sum_{v=1}^{n} \Re\left(1-\bar{z}^{\prime} z^{\prime}\right)^{-1}\right|=(n / 2)\left|p_{n}\left(z^{\prime}\right)\right|$ $=(n / 2) \max \left|p_{n}(z)\right|=n / 2$.

Another inequality for the derivative of a polynomial. Replacing the "Tchebychev deviation" by the "Bessel deviation," we obtain the following analog of the inequality formulated at the beginning of this paper.

Let $p_{n}(z)$ be a polynomial of degree $n$; then ${ }^{3}$

$$
\left\{(2 \pi)^{-1} \int_{-\pi}^{\pi}\left|p_{n}^{\prime}\left(e^{i \theta}\right)\right|^{2} d \theta\right\}^{1 / 2} \leqq n\left\{(2 \pi)^{-1} \int_{-\pi}^{\pi}\left|p_{n}\left(e^{i \theta}\right)\right|^{2} d \theta\right\}^{1 / 2} .
$$

Setting $p_{n}(z)=\sum_{p=0}^{n} \alpha_{\nu} z^{\nu}$, and using Parseval's formula, this inequality can be written in the form

which is obvious.

$$
\sum_{\nu=1}^{n}\left|\alpha_{\nu}\right|^{2} \nu^{2} \leqq n^{2} \sum_{\nu=0}^{n}\left|\alpha_{\nu}\right|^{2}
$$

We prove now the following analog of P. Erdös's conjecture.

If $\left|p_{n}(z)\right| \leqq 1$ holds as $|z| \leqq 1$, and $p_{n}(z) \neq 0$ for $|z|<1$, then in (6) the factor $n$ can be replaced by $2^{-1 / 2} n$. This factor is the best possible.

By Lemma II the following inequality holds:

$$
(2 \pi)^{-1} \int_{-\pi}^{\pi}\left|p_{n}^{\prime}\left(e^{i \theta}\right)\right|^{2} d \theta \leqq(2 \pi)^{-1} \int_{-\pi}^{\pi}\left|q_{n}^{\prime}\left(e^{i \theta}\right)\right|^{2} d \theta .
$$

Now, $q_{n}(z)=\sum_{\nu=0}^{n} \bar{\alpha}_{n-\nu} z^{\nu}$, hence by (7)

'See A. Zygmund, A remark on conjugate functions, Proc. London Math. Soc. (2) vol. 34 (1932) pp. 392-400, esp. pp. 394-396. 


$$
\begin{aligned}
\pi^{-1} \int_{-\pi}^{\pi}\left|p_{n}^{\prime}\left(e^{i \theta}\right)\right|^{2} d \theta & \leqq(2 \pi)^{-1} \int_{-\pi}^{\pi}\left|q_{n}^{\prime}\left(e^{i \theta}\right)\right|^{2} d \theta+(2 \pi)^{-1} \int_{-\pi}^{\pi}\left|p_{n}^{\prime}\left(e^{i \theta}\right)\right|^{2} d \theta \\
& =\sum_{\nu=0}^{n}\left(\nu^{2}+(n-\nu)^{2}\right)\left|\alpha_{\nu}\right|^{2} .
\end{aligned}
$$

The greatest of the numbers $\nu^{2}+(n-\nu)^{2}, \nu=1, \cdots, n$, is $n^{2}$. Therefore

$$
\pi^{-1} \int_{-\pi}^{\pi}\left|p_{n}^{\prime}\left(e^{i \theta}\right)\right|^{2} d \theta \leqq n^{2} \sum_{\nu=0}^{n}\left|\alpha_{\nu}\right|^{2}
$$

which was the assertion.

For $p_{n}(z)=\left(z^{n}+1\right) / 2$ the sign of equality holds in (8).

New York City

\section{A NEW FORMULA FOR INVERSE INTERPOLATION}

\section{HERBERT E. SALZER}

This paper is devoted to the derivation of a formula for inverse interpolation in a table of equally spaced arguments. The resulting formula (5) is more concise and convenient than those in existence. It involves neither differences nor polynomial coefficients other than small powers. In use it will be found much simpler and quicker than those given by Davis, Aitken, Steffensen and Milne-Thomson. In a sense, it is the analogue of the Lagrangian formula for direct interpolation without differences (that is, in terms of the tabular entries only) if the usual expression (right member of (1) below) is rearranged in terms of powers of the argument $p$.

Lagrange's general interpolation formula is

$$
f(x)=\sum_{\nu=0}^{\nu=k} \frac{P_{\nu}(x)}{P_{\nu}\left(a_{\nu}\right)} f\left(a_{\nu}\right), \quad \text { where } \quad P_{\nu}(x)=\frac{1}{\left(x-a_{\nu}\right)} \prod_{i=0}^{i=k}\left(x-a_{i}\right) .
$$

For equally spaced arguments at interval $h_{0}$ after suitable relabelling of the arguments $a_{i}$, Lagrange's formula becomes

$$
f_{p}=\sum_{i=-[(n-1) / 2]}^{i=[n / 2]} L_{i}^{(n)}(p) f_{i}
$$

Received by the editors January 1, 1944. 